\title{
Endomicrobial Community Profiles of Two Different Mealybugs: Paracoccus marginatus and Ferrisia virgata
}

\author{
Polpass Arul Jose ${ }^{1 \#}$, Ramasamy Krishnamoorthy ${ }^{1}$, Pandiyan Indira Gandhi ${ }^{2}$, \\ Murugaiyan Senthilkumar ${ }^{3}$, Veeranan Janahiraman', Karunandham Kumutha', \\ Aritra Roy Choudhury ${ }^{4}$, Sandipan Samaddar ${ }^{4 *}$, Rangasamy Anandham ${ }^{1 *}$, and Tongmin Sa ${ }^{4 *}$ \\ ${ }^{1}$ Department of Agricultural Microbiology, Agricultural College and Research Institute, Madurai, Tamil Nadu \\ Agricultural University, Tamil Nadu, India \\ ${ }^{2}$ Regional Research Station, Vridhachalam, Tamil Nadu Agricultural University, Tamil Nadu, India \\ ${ }^{3}$ Department of Agricultural Microbiology, Tamil Nadu Agricultural University, Coimbatore, Tamil Nadu, India \\ ${ }^{4}$ Department of Environmental and Biological Chemistry, Chungbuk National University, Cheongju 28644, \\ Republic of Korea
}

Mealybugs (Hemiptera: Coccomorpha: Pseudococcidae) harbor diverse microbial symbionts that play essential roles in host physiology, ecology, and evolution. In this study we aimed to reveal microbial communities associated with two different mealybugs, papaya mealybug (Paracoccus marginatus) and two-tailed mealybug (Ferrisia virgata) collected from the same host plant. Comparative analysis of microbial communities associated with these mealybugs revealed differences that appear to stem from phylogenetic associations and different nutritional requirements. This first report on both bacterial and fungal communities associated with these mealybugs provides a preliminary insight on factors affecting the endomicrobial communities.

Received: January 13, 2020 Accepted: March 31, 2020

First published online: April 02, 2020

*Corresponding authors

Phone: $+82-43-261-2561$ Fax: +82-43-271-5921 E-mail: tomsa@chungbuk.ac.kr R.A.

E-mail: anandhamranga@gmail.com

"Present address: Faculty of Agriculture, Food and Environment, Hebrew University of Jerusalem, POB 12, Rehovot 761000, Israel ${ }^{\ddagger}$ Present address: Department of Land, Air and Water Resources, University of California, Davis, California, USA

Supplementary data for this paper are available on-line only at http://jmb.or.kr.

pISSN 1017-7825 eISSN 1738-8872

Copyright(C) 2020 by The Korean Society for Microbiology and Biotechnology
Keywords: Ecology, mealybug, endomicrobiota, phylogeny, Paracoccus marginatus, Ferrisia virgata

Insects are the most abundant animals in terrestrial ecosystems and are inhabited by symbiotic microbes that provide beneficial services to their hosts [1]. Mealybugs (Homoptera: Coccoidea: Pseudococcidae) are plantsucking scale insects affecting agricultural ecosystems and causing damage to more than 300 plant species [2-4]. Examining the ecological interactions of symbiotic microbes in agriculturally important insect-hosts may lead to novel methods of pest control and enhancement of agricultural productivity $[5,6]$. Diet and the insect lineage have been proposed to exert different influences on symbiotic microbial diversity [7]. Accordingly, in this study we examined the hypothesis that the mealybug microbial ecology is strongly determined by interaction between phylogenetic constraints and nutritional requirements. To this end, we performed endomicrobial community analysis of two phylogenetically distinct mealybug species that feed concurrently on the same plant yet differ in their choice of feeding site and the processing of the plant-derived diet. 1) Papaya mealybug (PM), Paracoccus marginatus Williams and Granara de Willink, feeds frequently on phloem sap and produces large amounts of honeydew [8]. 2) Two-tailed mealybug (TM), Ferrisia virgata Cockerell, colonizes around the terminal parts of the plant, infrequently accesses the phloem sap and produces small amounts of honeydew [9]. Based on integrated molecular and morphological data, these two mealybugs have been assigned under different sub-families [10].

Pooled PM and TM samples were created from fifty individuals of each mealybug species collected from a papaya (Carica papaya) plant cv. CO8 at Agricultural College and Research Institute, Madurai $\left(9^{\circ} 56^{\prime} 0^{\prime \prime} \mathrm{N}\right.$ and $78^{\circ} 7^{\prime} 0 " \mathrm{E}$ ), India. The insects were surface sterilized by rinsing in sterile water, soaking in $70 \%$ ethanol and $10 \%$ bleach, with three rinses in water, for $60 \mathrm{sec}$ at each step. The surface-sterilized insects were homogenized in liquid nitrogen. Whole DNA was extracted from homogenates using an Insect DNA Purification Kit (Hi-Media, India) according to the manufacturer's protocol. The extracted DNA was amplified using primers targeting the V3-V4 region of the bacterial $16 \mathrm{~S}$ rRNA gene (primers, $341 \mathrm{~F}$ and 518R) and fungal internal transcribed spacer (primers, ITS5F and ITS2R) [11, 12]. Sequencing was performed using the Illumina MiSeq platform at Genotypic Technology, India. Resulted sequence data were deposited in SRA archives under accession number PRJNA522349. The raw reads were processed as described elsewhere [13]. The processing of raw reads clustered at $97 \%$ identity revealed 5,881 and 2,417 bacterial operational taxonomic units (OTUs), whereas 258 and 172 fungal OTUs were identified for PM and TM, respectively. The rarefaction curve (Fig. S1) indicated the adequate sampling effort for getting the full extent of taxonomic diversity. It explains how the number of species found in a sample at any given phylogenetic level is strongly affected by the number of sequences analyzed [14]. The Shannon 
Table 1. Microbial diversity estimates in the gut of papaya mealybug and two-tailed mealybug.

\begin{tabular}{cccccc}
\hline Domain & Type of mealybug & Number of OTUs & Chaol (Richness) & Shannon (Diversity) & Simpson (Diversity) \\
\hline \multirow{2}{*}{ Bacteria } & PM & 5881 & 10061.46 & 3.42 & 0.64 \\
& TM & 2417 & 4833.76 & 1.87 & 0.48 \\
\multirow{2}{*}{ Fungi } & PM & 258 & 276 & 1.30 & 0.91 \\
& TM & 170 & 172 & 0.54 & 0.48 \\
\hline
\end{tabular}

and Simpson diversity indices, and richness estimator (Chao) of bacteria and fungi were relatively higher for PM compared to TM (Table 1). However, the diversity and richness indices of fungi were relatively poor for both the samples compared to the bacterial counterpart.

The gut bacterial community had proteobacterial abundance for both of the mealybug species, followed by Actinobacteria and Firmicutes in PM and TM, respectively (Fig. 1A). A previous study of 305 individual insects belonging to 21 taxonomic orders showed that the insect microbiota is dominated by the Proteobacteria and

A

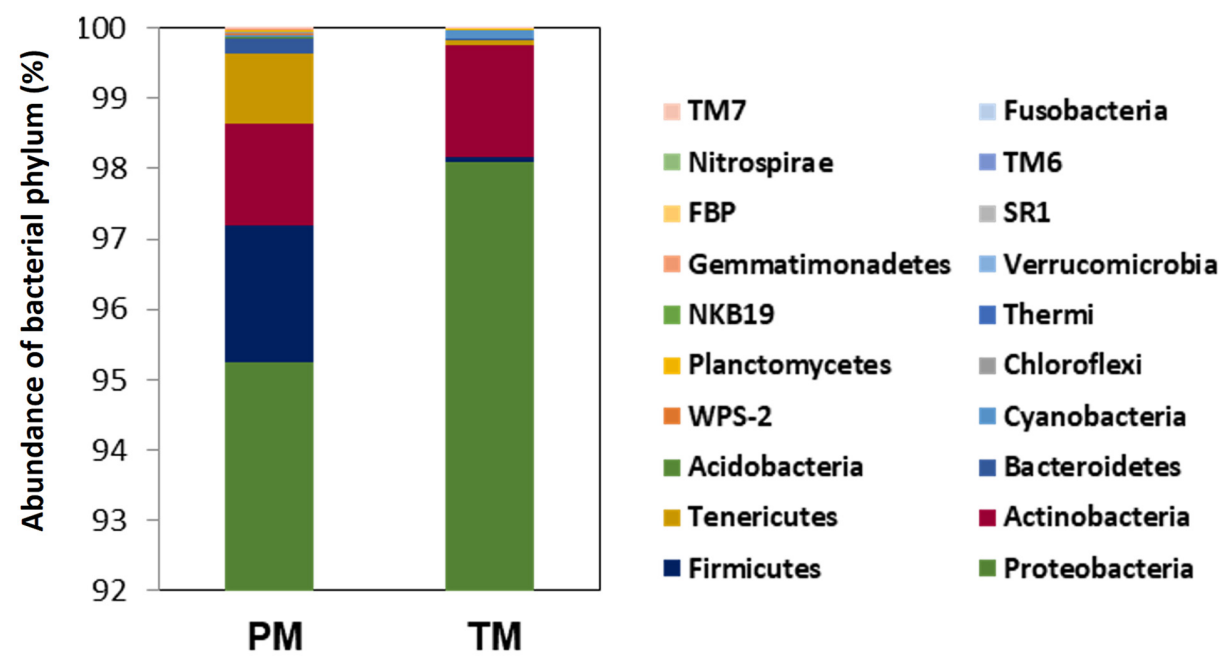

B

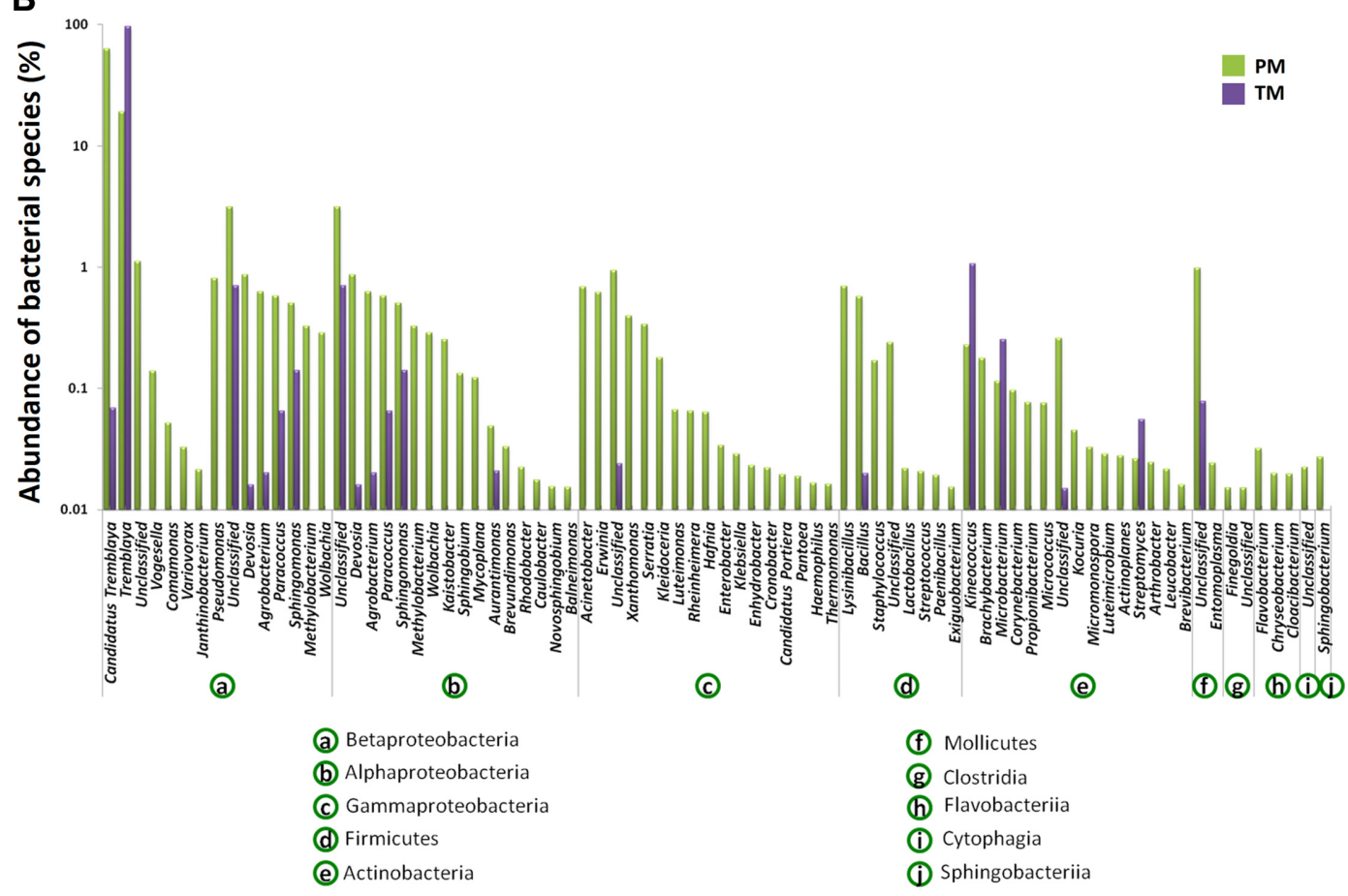

Fig. 1. Bacterial community profile decoded by $16 \mathrm{~S}$ amplicon-metagenomics. Relative abundance of the gutbacterial lineages (after removing endosymbionts) found in PM and TM at (A) phylum level and (B) species level. 
Firmicutes [15]. Moreover, the specific abundance of Proteobacteria further correlates well with bacterial communities of different mealybug species [16-18]. Recently, Iasur-Kruh et al. [17] reported that Betaproteobacteria (58\%) and Gammaproteobacteria (33\%) constituted the proteobacterial community in the vine mealybug. However, this pattern deviated in papaya and two-tailed mealybugs. In papaya mealybug, all the three classes of Proteobacteria (Betaproteobacteria (83.41\%), Alphaproteobacteria (7.19\%) and Gammaproteobacteria (4.63\%) were represented, while Betaproteobacteria is most abundant $(97.6 \%)$ in two-tailed mealybug (Fig. 1B). At the genus level, PM and TM samples were dominated by the obligate nutritional endosymbionts Tremblaya, which has been reported to provide aid in nutrition and detoxification of plant substances $[19,20]$. The ITS sequencebased fungal community analysis revealed the occurrence of seven fungal phyla (Fig. 2A). The majority of sequences were affiliated with Ascomycota (78.75\%), followed by Zygomycota (4.74\%) and Basidomycota (5.91\%). Individually, Ascomycota dominated with $75.28 \%$ and $91.65 \%$ in PM and TM respectively. The phylum Zygomycota and two different unclassified populations were moderately abundant in PM, while it was less abundant in TM. However, Glomeromycota was more abundant in TM rather than in PM. However, among the classified genera, Cladosporium showed moderate abundance (8.04\%), followed by Aureobasidium (5.54\%), Mortierella (4.61\%). Notably, the majority of Aureobasidium and Mortierella counts were from PM (Fig. 2B). The results of earlier studies using culture-dependent and molecular techniques indicated that vine mealybug, Planococcus ficus, largely harbors Ascomycota [18]. Cladosporium and Mycosphaerella, common environmental fungi, were equally found in both TM and PM. Iasur-Kruh et al. [17] found that Cladosporium is abundant in vine mealybug reared in lab. This suggests that Cladosporium and Mycosphaerella are more transient fungal associates

A

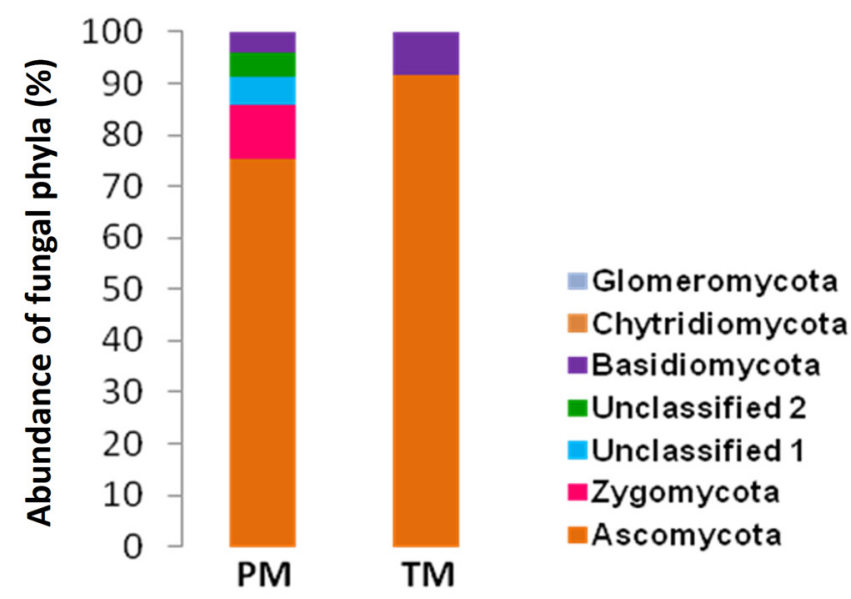

B

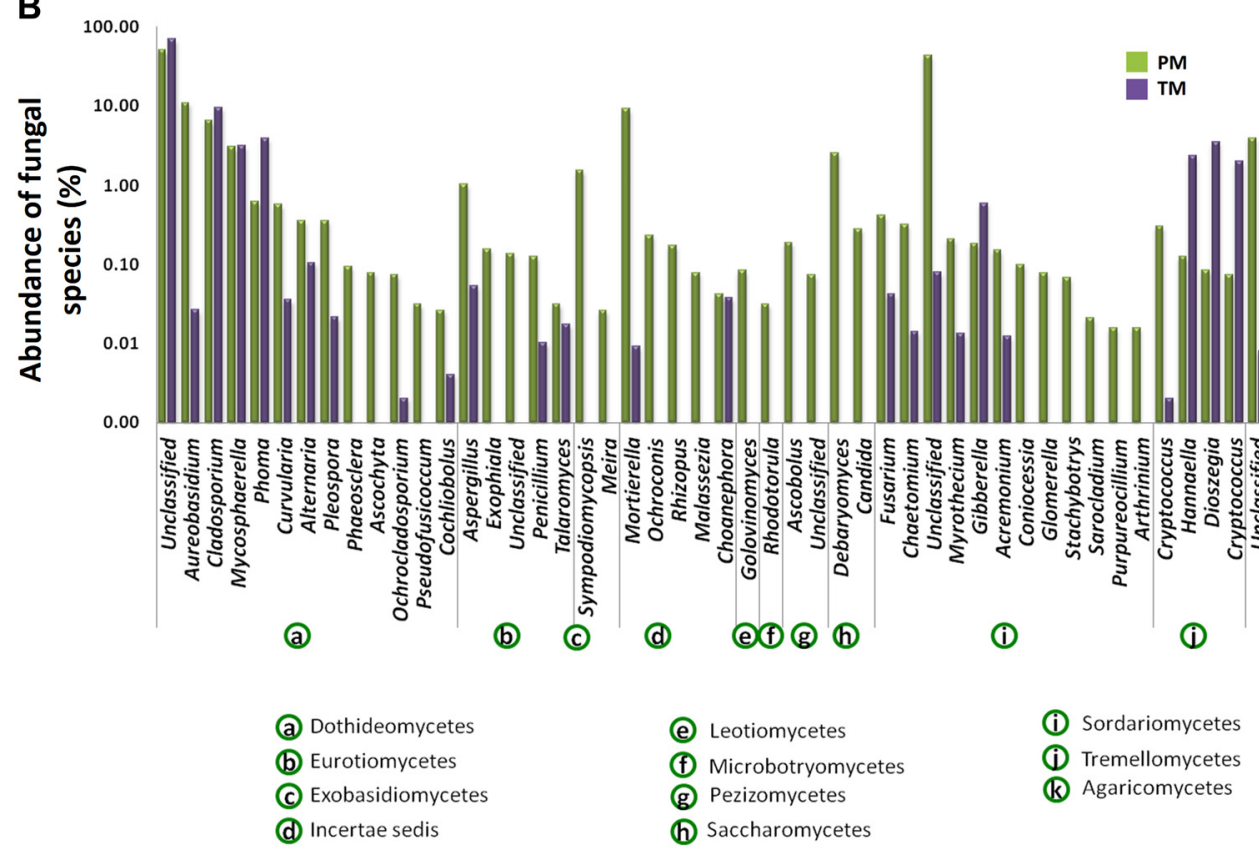

Fig. 2. Fungal community profile decoded by $18 S$ (ITS) amplicon-metagenomics. Relative abundance of the gutfungal lineages (after removing endosymbionts) found in PM and TM at (A) phylum level and (B) species level. 


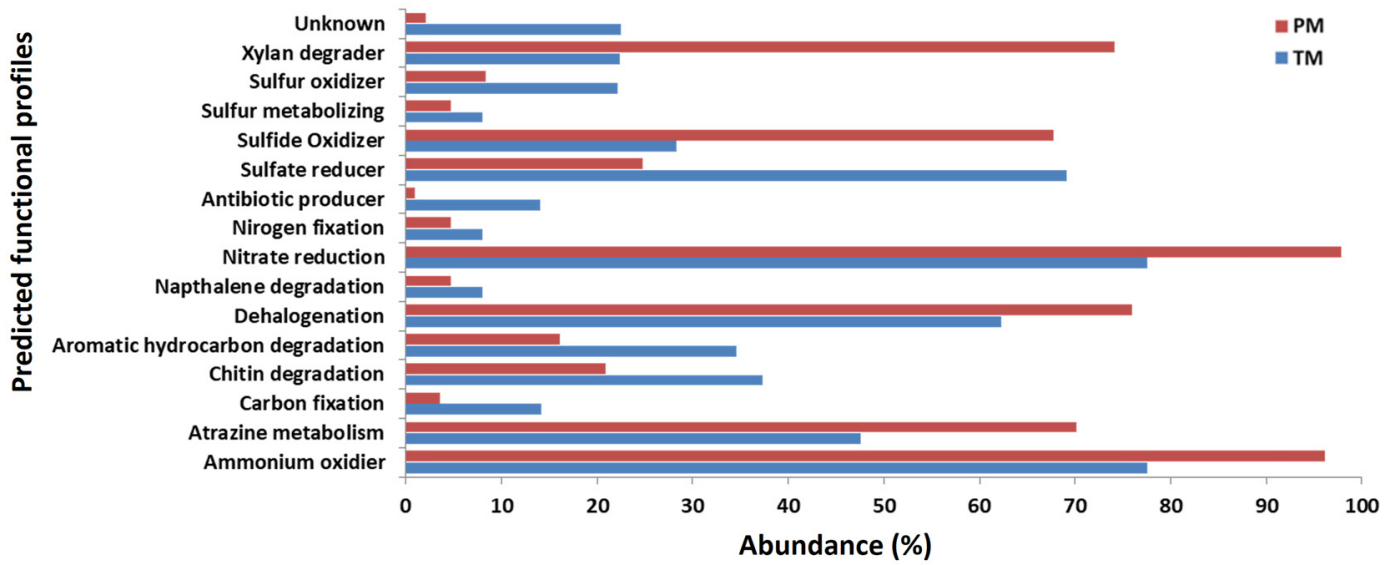

Fig. 3. Functional mapping of the bacterial community associated with PM and TM.

acquired via feeding. A recent study using confocal microscopy revealed that a similar fungus, Beauveria bassiana, penetrates mealybug Phenacoccus manihoti through the legs and mouthparts [21]. Interestingly, several fungal species were found in one mealybug species, while not in the other, though they were sampled from the same plant. For instance, Zygomycota was found in PM, but not in TM. Similarly, Glomeromycota was found in TM, yet it is absent in PM. These differences could be due to selective ecological pressure exerted by the host $[18,22]$.

Among the symbionts associated with PM and TM, Tremblaya is a well-known endosymbiont of mealybugs. Bacterial communities other than Tremblaya associated with the PM and TM were retrieved from the taxonomy data and mapped for their metabolic activities using different phenotypic categories in METAGENassist [23]. The analysis was done using the standard settings suggested in the server. The results revealed the occurrence of 15 types of metabolic activities (Fig. 3). Interestingly, abundant bacteria capable of ammonia oxidization, atrazine metabolism, dehalogenation, nitrate reduction, sulfur metabolism, and xylan degradation were found in both mealybug species. The automated metabolic functional mapping of the abundant bacterial community revealed diverse activities, and strongly suggested the role of the bacterial communities associated with both mealybugs in providing nutritional as well as detoxification support to the host insects. For instance, those microbes having the ability to fix and mobilize different nutrients (nitrogen and sulfur) could provide dietary support to the insect host, while others are involved in detoxification of toxic compounds [24]. Difference in the relative abundance of different metabolic functions suggests evolutionary trajectories of the microbial communities, tailored to specific needs of the hosts [24,25].

In summary, this study disclosed the endomicrobial communities (bacteria and fungi) in two mealybug species, P. marginatus and F. Virgata, differ in phylogenetic and nutritional characteristics. Despite a lack of sequencing replicates, this study provides a preliminary insight into the relationships between different mealybugs and their microbial communities. Differences among the microbial communities appear to be associated largely with their phylogeny and different nutritional characteristics while they fed on the same plant sap. The super dominant bacteria was Tremblaya in both cases, but intra generic diversity was found within this genus in PM. Dynamics of Tremblaya in PM, as well as the moderately abundant and rare species in both mealybug species have to be studied under controlled conditions to reveal their biological and symbiotic roles.

\section{Availability of Data and Materials}

The data and analyses from the current study are available from the corresponding author upon reasonable request. The raw reads were deposited in SRA archives and can be accessed by accession number PRJNA522349.

\section{Acknowledgments}

This study was supported by the Rapid Grant for Young Investigator (RGYI), Department of Biotechnology (DBT), Ministry of Science and Technology, Government of India under grant no. BT/PR6430/GBD/27/412/ 2012. The authors are so grateful to Prof. Boaz Yuval for his critical reading of the manuscript.

\section{Conflict of Interest}

The authors have no financial conflicts of interest to declare.

\section{References}

1. Moran NA, McCutcheon JP, Nakabachi A. 2008. Genomics and evolution of heritable bacterial symbionts. Annu. Rev. Genet. 42: 165190.

2. Miller DR, Miller GL. 2002. Redescription of Paracoccus marginatus Williams and Granara de Willink (Hemiptera: Coccoidea: Pseudococcidae), including descriptions of the immature stages and adult male. Proc. Entomol. Soc. Wash. 104: 1-23. 
3. Amarasekare KG, Mannion CM, Osborne LS, Epsky ND. 2014. Life history of Paracoccus marginatus (Hemiptera: Pseudococcidae) on four host plant species under laboratory conditions. Environ. Entomol. 37: 630-635.

4. Normark BB, Johnson NA. 2011. Niche explosion. Genetica 139: 551-564.

5. Zindel R, Gottlieb Y, Aebi A. 2011. Arthropod symbioses: a neglected parameter in pest-and disease-control programmes. J. Appl. Ecol. 48: 864-872.

6. Yuval B, Ben-Ami E, Behar A, Ben-Yosef M, Jurkevitch E. 2013. The Mediterranean fruit fly and its bacteria - potential for improving sterile insect technique operations. J. Appl. Entomol. 137: 39-42.

7. Yun JH. 2014. Insect gut bacterial diversity determined by environmental habitat, diet, developmental stage, and phylogeny of host. Appl. Environ. Microbiol. 80: 5254-5264.

8. Miller DR, Williams DJ, Hamon AB, 1999. Notes on a new mealybug (Hemiptera: Coccoidea: Pseudococcidae) pest in Florida and the Caribbean: the papaya mealybug, Paracoccus marginatus Williams and Granara de Willink. Insecta Mundi 13: 179-181

9. Kaydan MB, Gullan J. 2012. A taxonomic revision of the mealybug genus Ferrisia fullaway (Hemiptera: Pseudococcidae) with descriptions of eight new species and a new genus. Zootaxa 3543: 1.

10. Hardy NB, Gullan PJ, Hodgson CJ. 2008. A subfamily-level classification of mealybugs (Hemiptera: Pseudococcidae) based on integrated molecular and morphological data. Syst. Entomol. 33: 51-71.

11. Whiteley AS, Jenkins S, Waite I, Kresoje N, Payne H, Mullan B, et al. 2012. Microbial 16S rRNA Ion Tag and community metagenome sequencing using the Ion Torrent (PGM) Platform. J. Microbiol. Methods 91: 80-88

12. Shen Y, Nie J, Li Z, Li H, Wu Y, Dong Y, Zhang J. 2018. Differentiated surface fungal communities at point of harvest on apple fruits from rural and peri-urban orchards. Sci. Rep. 8: 2165.

13. Niu B, Fu L, Sun S, Li W. 2010. Artificial and natural duplicates in pyrosequencing reads of metagenomic data. BMC Bioinformatics 11: 187.

14. Schloss PD, Handelsman J. 2005. Introducing DOTUR, a computer program for defining operational taxonomic units and estimating species richness. Appl. Environ. Microbiol. 71: 1501-1506.

15. Yun JH. 2014. Insect gut bacterial diversity determined by environmental habitat, diet, developmental stage, and phylogeny of host. Appl. Environ. Microbiol. 80: 5254-5264.

16. Gruwell ME, Hardy NB, Gullan PJ, Dittmar K. 2010. Evolutionary relationships among primary endosymbionts of the mealybug subfamily Phenacoccinae (Hemiptera: Coccoidea: Pseudococcidae). Appl. Environ. Microbiol. 76: 7521-7525.

17. Iasur-Kruh L, Taha-Salaime L, Robinson WE, Sharon R, Droby S, Perlman SJ, et al. 2015. Microbial associates of the vine mealybug Planococcus ficus (Hemiptera: Pseudococcidae) under different rearing conditions. Microb. Ecol. 69: 204-214.

18. Ivens AB, Gadau A, Kiers ET, Kronauer DJ. 2018. Can social partnerships influence the microbiome? Insights from ant farmers and their trophobiont mutualists. Mol. Ecol. 27: 1898-1914.

19. Buchner P. 1965. Endosymbiosis of animals with plant microorganisms. pp. 168. USA: Interscience Publishers. (No. QH548 B743)

20. McCutcheon JP, von Dohlen CD. 2011. An interdependent metabolic patchwork in the nested symbiosis of mealybugs. Curr. Biol. 21: $1366-1372$.

21. Amnuaykanjanasin A, Jirakkakul J, Panyasiri C, Panyarakkit P, Nounurai P, Chantasingh D. 2013. Infection and colonization of tissues of the aphid Myzus persicae and cassava mealybug Phenacoccus manihoti by the fungus Beauveria bassiana. BioControl 58: 379-391.

22. Gomez-Polo P, Ballinger MJ, Lalzar M, Malik A, Ben-Dov Y, Mozes-Daube N. 2017. An exceptional family: Ophiocordyceps-allied fungus dominates the microbiome of soft scale insects (Hemiptera: Sternorrhyncha: Coccidae). Mol. Ecol. 26: 5855-5868.

23. Arndt D, Xia J, Liu Y, Zhou Y, Guo AC, Cruz JA, et al. 2012. METAGENassist: a comprehensive web server for comparative metagenomics. Nucleic Acids Res. 40: W88-W95.

24. Douglas AE. 2009. The microbial dimension in insect nutritional ecology. Funct. Ecol. 23: 38-47.

25. Flórez LV, Biedermann PH, Engl T, Kaltenpoth M. 2015. Defensive symbioses of animals with prokaryotic and eukaryotic microorganisms. Nat. Prod. Rep. 32: 904-936. 